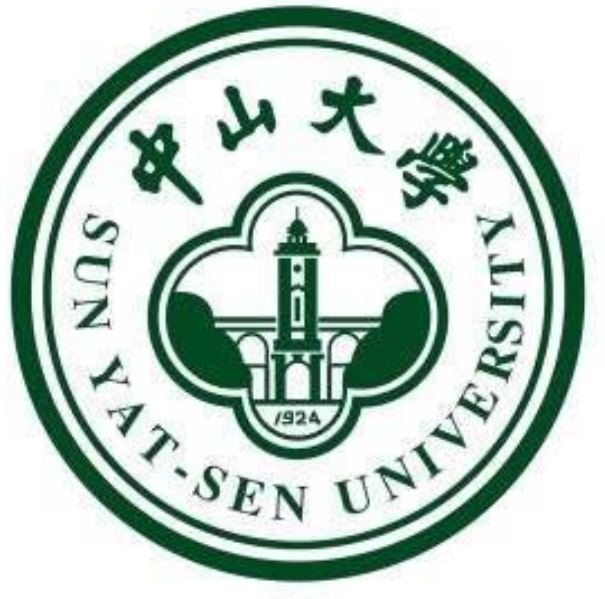

\title{
Anti-inflammatory effect of DHA in mice submitted to acute ischemic stroke
}

Sanxin $\mathrm{Liu}^{1}$, Wei Cai ${ }^{1}$, Mengyan $\mathrm{Hu}^{1}$, Zhengqi $\mathrm{Lu}^{1 *}$

${ }^{1}$ Department of Neurology, The Third Affiliated Hospital of Sun Yat-sen University, Guangzhou, China

*Corresponding author

\section{INTRODUCTION}

Ischemic stroke is a major cause of death worldwide and responsible for serious long-time disability in adults. It was concluded that DHA has the potential for treating ischemic stroke, but the mechanism is unclear. The study aimed to investigate the antiinflammatory effect of DHA in mice suffering from acute cerebral ischemia/ reperfusion injury.

\section{METHODS}

- Cerebral ischemia in mice was induced by middle cerebral artery occlusion (MCAO) for $1 \mathrm{~h}$.

- WT C57/BL6 mice submitted to tMCAO were treated with DHA $(10 \mathrm{mg} / \mathrm{kg}$, every 24 h, i.p. $3 d$ ) or Vehicle (Veh, saline).

- Stroke outcomes, systemic inflammatory status and microglia/macrophage phenotypic alterations were assessed 3 days after stroke.

- Macrophage depletions were induced by clodronate liposomes injection.

- Primary macrophage cultures were used to evaluate the direct effect of DHA on macrophages.

\section{Figure 1}

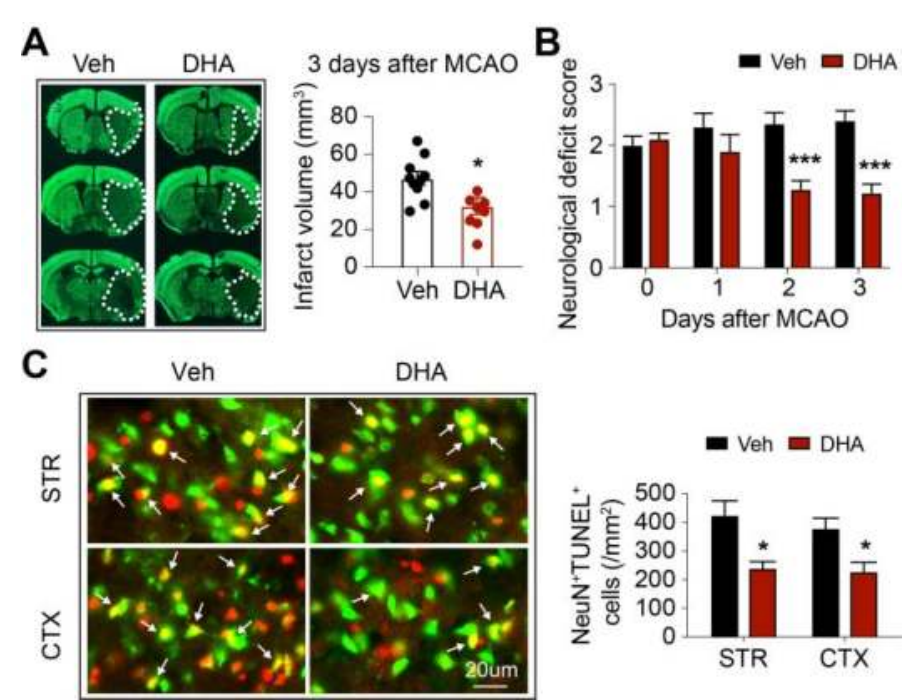

Fig.1 Post-stroke DHA treatment protects against acute cerebral ischemia. C57/BL6 mice were subjected to 60 min $\mathrm{MCAO}$ followed by daily DHA $(10 \mathrm{mg} / \mathrm{kg}$, i.p.) or saline (Veh, vehicle) treatment for 3 days. A) Infarct volume was quantified on NeuN (green)-stained coronal sections. Neurological deficit score was assessed at day 1-3 after IMCAO C) Left: Representative images demonstrating TUNEL (red) co-labeling with NeuN (green) in infarct penumbra 3 days after MCAO. Right: Quantification of the number of NeuN+TUNEL+ neurons. $N=4{ }^{*} \mathrm{P} \leq 0,05,{ }^{* * *} \mathrm{P} \leq 0.001$.

\section{Figure 3}

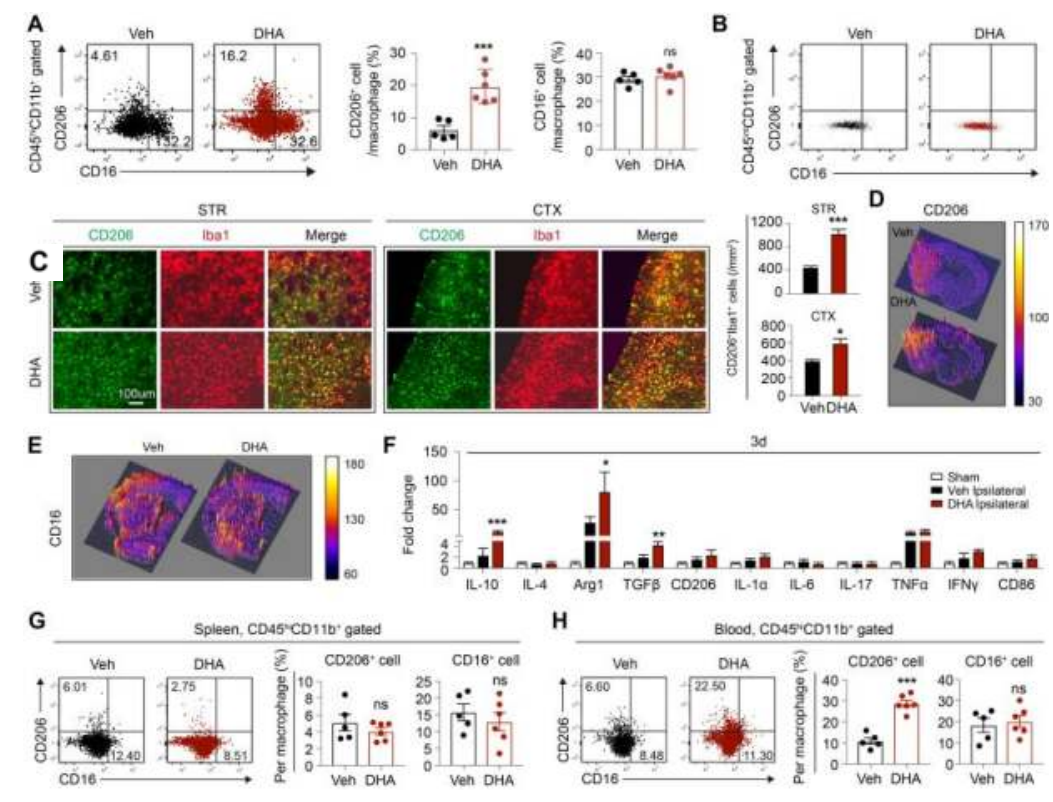

Fig.3 Post-stroke DHA therapy induces anti-inflammatory phenotype of macrophages in the ischemic brain. A) Representative FACS panels showing that DHA treatment induced an anti-inflammatory phenotype of macrophages. B) Representative FACS panels showing that microglia expressed low levels of CD206 and CD16. C) Immunofluorescent staining analysis of CD206 and lba1. $\mathrm{N}=4$. D-E) 3D reconstructed plot showing CD206Alexa Fluor 488 and CD16-Alexa Fluor 488 fluorescence intensity. F) mRNA with RT-PCR in the ipsilateral brain 3 days after tMCAO. $N=3$. ${ }^{*} P \leq 0.05$, ${ }^{* *} P \leq 0.01,{ }^{* * *} P \leq 0.001$. G-H) Representative FACS panels and quantification in blood (D) and spleen (C). $N=5$. ${ }^{* * *} \mathrm{P} \leq 0.001$

\section{Figure 5}

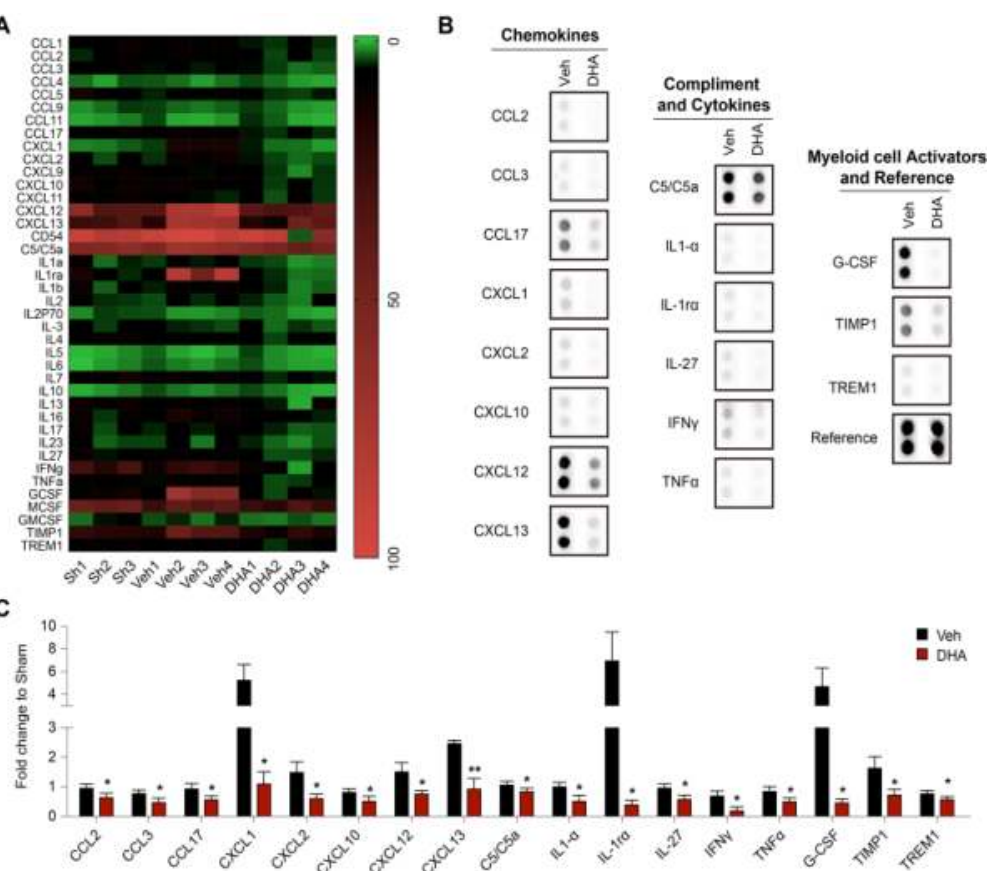

Fig.5 Systemic DHA injection reduces circulating inflammatory factors after ischemic stroke. Cytokine and chemokine production were measured in blood using protein array 3 days after IMCAO. Heat map (A), representative do plots (B) and quantification (C) showed that post-stroke DHA treatment downregulates chemokines, complement, cytokines and myeloid cell activators in blood 3 days after $\mathrm{MCAO} . \mathrm{N}=3$. ${ }^{*} \mathrm{P} \leq 0.05,{ }^{* *} \mathrm{P} \leq 0.01$.

\section{Figure 2}

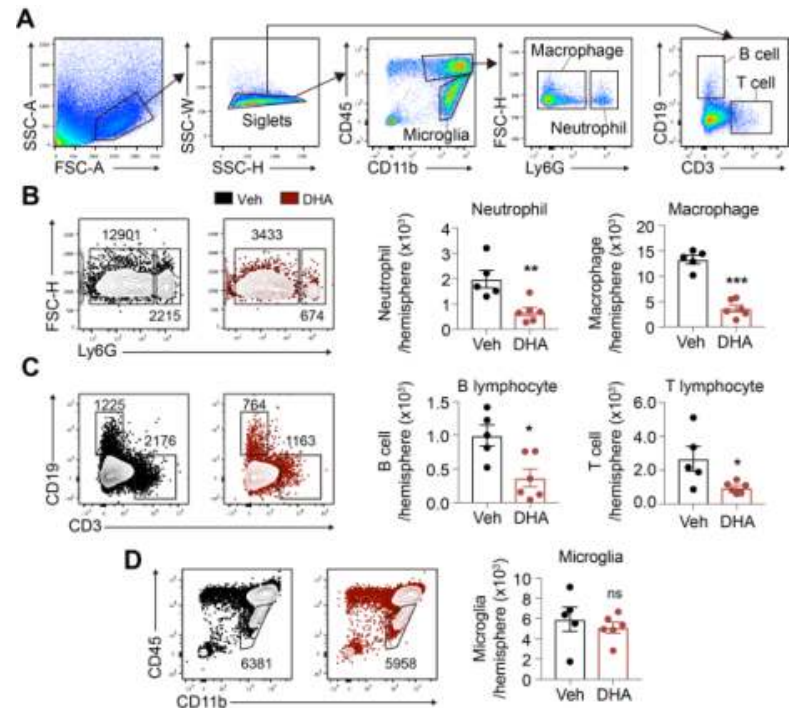

Fig.2 Systemic DHA therapy reduces immune cell infiltration into the (a) was analyzed by flow cytometry 3 days after IMCAO. A) Gating strategy for alyzed by flow cylometry 3 days ater $M C A O$. A) Gating strategy lymphocytes. B-D) Representative FACS panels and quantification of the numbers of immune cells in the ipsilateral brain $N=5{ }^{*} P \leq 0.05,{ }^{* *} P \leq 0.01$ ${ }_{* * *} \leq 000$

\section{Figure 4}

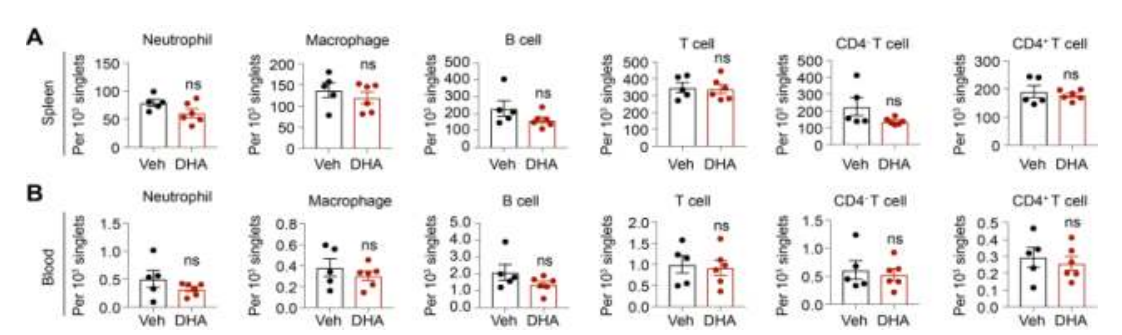

Fig.4 Systemic DHA injection shifts circulating macrophag polarity toward anti-inflammatory M2 phenotype after ischemic stroke without changing the immune cell numbers in blood and spleen. A-B) Immune cells in spleen (A) and blood (B) were assessed with flow cytometry 3 days after tMCAO. $N=5$.

\section{Figure 6}

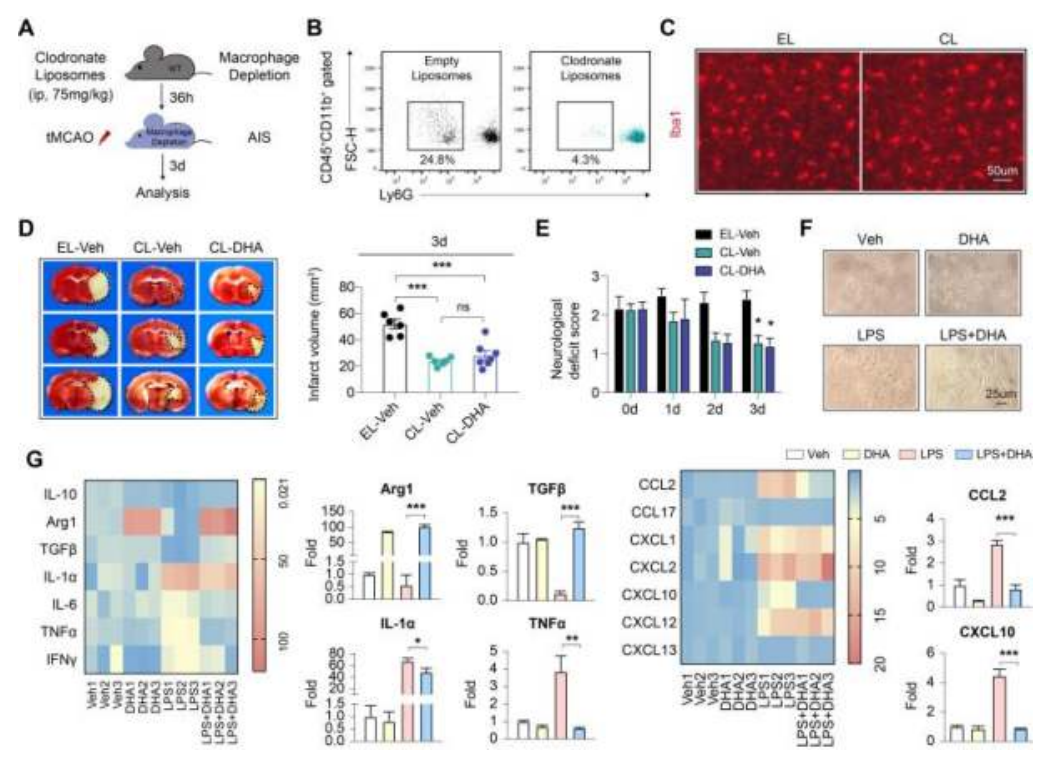

Fig.6 Macrophages are essential for the neuroprotective effects of systemic DHA administration. A) Timeline of Clodronate liposomes application and stroke outcome analysis. Macrophages were depleted with Clodronate liposomes. B-C) Impact of Clodronate liposomes on blood macrophages (B) and brain microglia (C) in sham mice 36 hours fter liposome application. $\mathrm{N}=3$. D) Infarct volume was assessed with TTC staining 3 days after stroke. $N=6$. E) Neurological deficit score was assessed at 0-3 days after stroke. $\mathrm{N}=6$. F) Representative images showing morphological alteration of macrophages after LPS activation with or without DHA treatment. (G) mRNA expression of inflammatory mediators in activated macrophages were measured by RT-PCR. ${ }^{*} P \leq 0.05,{ }^{* *} P \leq 0.01,{ }^{* * *} P \leq 0.001$

\section{CONCLUSION}

- Post stroke DHA treatment decreased infarct volume significantly compared with control group

- Post stroke DHA treatment induces inflammatory resolving phenotype of macrophage after cerebral ischemia

- Post stroke DHA treatment down-regulates chemokines and other pro-inflammatory markers

- Post-stroke DHA treatment ameliorated acute ischemic brain injury in macrophage-dependent manner 